\title{
Comunicación en el gobierno: lo gubernamental y lo público en la transformación de la relación con el ciudadano
}

\section{Communication in government: the governmental and the public in the transformation of citizen relationship}

Karla Negrete Huelga*

Universidad Autónoma de Querétaro

Facultad de Ciencias Políticas y Sociales

Cerro de las campanas $s / n$, Las campanas, C.P. 76010, Santiago de

Querétaro, Querétaro, México

Editor: Rogelio del Prado Flores

Fecha de recepción: 23 de septiembre de 2021

Fecha de aceptación: 3 de diciembre de 2021 karla.negrete@uaq.mx

https://orcid.org/0000-0003-3483-783X

RESUMEN

El desarrollo de la democracia supone cambios en la relación entre ciudadanos y gobernantes. Esto conlleva, a su vez, a transformaciones en los procesos de comunicación desde los gobiernos, que se ven en la necesidad de adoptar nuevos paradigmas. El presente artículo tiene como objetivo hacer una revisión de las formas de abordaje de la comunicación desde las instancias gubernamentales. Mediante una revisión documental, se revisan las lógicas de comunicación desde el gobierno, centrándose en la comunicación gubernamental y la comunicación pública como dos abordajes vinculados a la democracia y el ámbito político. Así, se muestra que tanto la comunicación gubernamental, como la comunicación pública son necesarias para los gobiernos; sin embargo, es la de carácter público la que conlleva un mayor acercamiento con el ciudadano.

Palabras clave: democracia, comunicación gubernamental, comunicación pública.

\footnotetext{
* Licenciada en Comunicación y Periodismo y Maestra en Comunicación y Cultura Digital por la Universidad Autónoma de Querétaro. Coordinadora Técnica del laboratorio "LabUAQ Ciudadanía Digital” de la Universidad Autónoma de Querétaro. Reconocimiento al Mérito Académico 2017 por la Universidad Autónoma de Querétaro. Publicaciones recientes en la Revista Mexicana de Opinión Pública (2020) y Cuadernos.info (2018) sobre temas de gobierno abierto, comunicación política y entornos digitales.
} 


\section{ABSTRACT}

Democracy development involves changes in the relationship between citizens and governing authorities. This leads, in turn, to transformations in the communication processes from governments, which needs to adopt new paradigms. The aim of this article is to review the ways of approaching communication from government instances. Through a documentary review, the logics of communication from the government are reviewed, focusing on government communication and public communication as two approaches linked to democracy and the political sphere. Thus, it's shown that both, government communication and public communication are vecessaru for governmets; however, it's the public nature that entails a greater approachment with citizens.

Keywords: citizen participation, digital environments, political communication.

\section{INTRODUCCIÓN}

En la sociedad de la información el progreso, la competitividad y la tecnología son elementos dinámicos que inciden en las esferas económicas, políticas y sociales. En el ámbito político, las transformaciones también se encuentran presentes. En un principio, las instancias gubernamentales, regidas por la democracia representativa, se encontraban desconectadas de las demandas ciudadanas, por lo que la gestión pública se enfrenta a entornos más complejos en los que surgen redes ciudadanas e intermediarios digitales (Villodre, 2019). Por tanto, la organización y la gestión gubernamental evolucionan de esquemas cerrados a otros más abiertos y flexibles, que reflejan la necesidad de un papel más activo por parte de la ciudadanía.

En un contexto de desconfianza y apatía ciudadana, la resignificación de la democracia es un imperativo. La innovación se convierte, así, en un elemento necesario para la transición democrática, por lo que no hacer cambios en la gestión gubernamental para hacer frente a las nuevas demandas ciudadanas puede tener consecuencias negativas. Asílo define Castells (1997):

Aquellos Gobiernos, o partidos, que no entiendan la nueva forma de hacer política y que se aferren a reflejos estatistas trasnochados, serán simplemente superados por el poder de los flujos y borrados del mapa político por los ciudadanos, tan pronto su ineficiencia y su parasitismo social sea puesto de manifiesto por la experiencia cotidiana. (párr. 6) 
La comunicación desde el ámbito gubernamental también requiere de cambios y resignificaciones, que vayan a la par con la transición democrática y el proceso de apertura. En dicho contexto, el presente artículo tiene como objetivo hacer una revisión de las formas de abordaje de la comunicación desde las instancias gubernamentales. Mediante una revisión documental, se busca realizar un recorrido por las aproximaciones de la comunicación que acompañan al quehacer gubernamental, centrándose en las características de la comunicación gubernamental y la comunicación pública, para reconocer si estos cambios han proporcionado una mejor relación entre la sociedad y el gobierno, como parte de los esquemas para combatir la desafección ciudadana.

\section{MARCO CONTEXTUAL}

La formación de la sociedad civil como un proceso ha generado reflexiones desde los debates filosóficos en la antigua Grecia, hasta los tiempos actuales. Algunos lo ven como un proceso lineal, otros lo divisan como algo desordenado, imparcial (Monterroso, 2009). Foucault (1979) apuesta por una visión mucho más amplia desde la genealogía, como una forma de saber minuciosa opuesta al despliegue de significaciones ideales de la metahistoria. El desarrollo no es el mismo en cada lugar, algunas regiones obtienen un mayor avance, otras quedan estancadas (Schumpeter, I943). No obstante, todas tienen un reto en común: las desigualdades sociales.

Para Rawls (197I) aunque la estructura pueda llegar a presentar injusticias, los principios de eficiencia que rigen a la sociedad autorizan cambios "que pudieran reducir las expectativas de algunos de los mejor situados” (p. 84). De ahí que las propuestas de Rawls (I97I) visibilicen un panorama en donde los ideales de desarrollo tienen cabida, sin dejar de lado los principios de diferencia entre individuos, los cuales son clave para la democracia. De acuerdo con este principio, se defiende la libertad de las personas de tener o no una vida con mayores o menores ventajas, que son parte de su naturaleza (nacer en una familia adinerada o en una familia con pocas oportunidades); lo que necesita hacer la sociedad es lograr que los menos desafortunados puedan prosperar, sin que esto signifique que los más aventajados no lo puedan hacer. Así se muestra que, bajo los principios democráticos, el bienestar social mantiene a todos dentro de sus objetivos, por lo que las políticas y acciones buscarán beneficiar a todos.

Desde las antiguas aportaciones sobre la democracia y su relación con los principios de la justica, hasta los tiempos actuales, el término ha sido parte de una discusión como parte del proceso constructivo de las sociedades. En un principio la noción de la democracia se centra en una manera representativa de gobernar, donde los ciudadanos tienen un papel 
secundario (Pateman, 1970). En este sentido, Schumpeter (1943) percibe a los ciudadanos como una masa electoral, que "es incapaz de una acción que no sea una estampida" (p. 283). De acuerdo con esta visión, para que la democracia funcione se necesita que suficientes ciudadanos participen en los procesos electorales, únicamente bajo una participación basada en la elección de representantes políticos; de esta forma, las visiones clásicas de la democracia no contemplan la participación, incluso visibilizan en esta un peligro: "Los participantes podrían alterar la estabilidad del sistema democrático” (Pateman, I970, p. 3).

Los cambios de paradigma en torno a la democracia no se desarrollaron de forma aislada. En un entorno donde el poder del Estado se debilita, surgen pensadores que comienzan a cuestionar las formas de gobernar. Con la evolución de la sociedad se ofertan nuevas visiones para la comprensión del ciudadano, quien no solo vota de manera masiva, sino que actúa bajo predisposiciones y actitudes que jugarán un papel importante en la decisión de voto. En un panorama de apatía ciudadana, las concepciones posteriores a la teoría clásica de la democracia apuestan por el conocimiento de las experiencias con la autoridad para conocer cómo moldean las personalidades de los ciudadanos (Pateman, 1970). En ellas se muestra, además, la importancia de educar al electorado para hacer votaciones socialmente responsables. Habermas (1994) enfatiza en adquirir una mayor capacidad de razonamiento a partir de los flujos de información, para lograr un papel más activo en los asuntos públicos. El autor habla al respecto desde la publicidad institucionalizada por el Estado como un elemento que busca influir en los espacios íntimos de la sociedad; no obstante, reconoce que cuando hay una capacidad racional se logra la construcción de ideologías propias. Así, la información fluye y aparecen sistemas mediadores, como los medios de comunicación, que poseen el poder de vigilar y hacer valer la rendición de cuentas. La apertura de la información logra un efecto en el que los medios, de acuerdo con Habermas (1994), ofrecen a la población la capacidad de razonar. En el contexto de la creación de lo público, Habermas (I994) menciona que:

la abolición de la institución de la censura previa significa una nueva etapa en el desarrollo de la publicidad; posibilita la penetración del razonamiento en la prensa, permitiendo que ésta se desarrolle hasta convertirse en un instrumento que impele a que las decisiones políticas sean tomadas ante la nueva tribuna del público. (p. 95)

En el contexto actual, la concepción de la democracia cambia. Los síntomas de una crisis de los Estados-nación son visibles: la apatía de los ciudadanos, la falta de legitimidad y el debilitamiento del poder derivado del neoliberalismo son algunas de sus manifestaciones. Atravesados por la globalización política, el discurso internacional se caracteriza por hablar de gobernanza, innovación y apertura a otras filosofías democráticas, para "explorar 
y potenciar formas de organización social que favorezcan la reconstrucción de vínculos, la articulación de sentidos colectivos de pertenencia respetuosos con la autonomía individual" (Subirats, 2007, p. 30). Así, surgen nuevas concepciones que parten de la participación como un principio democrático. La democracia participativa se puede legitimar cuando las decisiones emanen de una completa y abierta deliberación entre sus grupos, órganos y representantes; de ahí que busque "recuperar la voz, la presencia y los saberes de los que han ido siendo apartados de los ámbitos decisionales, reforzando las capacidades educativas de la participación, ya que exige entender los propios intereses y los de los demás" (Subirats, 2007, p. 35). A estas aportaciones, Zallo (2016) agrega otros puntos a cumplir para caracterizar a la democracia participativa: el respeto a la libertad, la justicia y los derechos humanos, el trato ecuánime a los grupos sociales y la toma de decisiones justas. Al dar voz a los ciudadanos se logra una toma de decisiones colectivas y nuevas formas de organización, que dejan ver un papel del Estado más abierto a la deliberación; es así como la colaboración se vuelve imprescindible para dar legitimidad a las decisiones políticas, renovar la confianza de los ciudadanos, responsabilizarlos de la toma de decisiones, y estimular la democracia y la ciudadanía activa (Aguirre Sala, 2012).

Los cambios en la democracia conllevan también transformaciones en las interacciones entre ciudadanos y sus gobernantes. Es ahí donde se inserta la comunicación como un elemento que se desarrolla a la par de la democracia "porque la comunicación es un elemento cada vez más central para la democracia, se torna imprescindible que existan mecanismos sociales para garantizar una mayor democracia de la comunicación” (Galvis y Botero, 2013, p. 29). Esto se debe a la posibilidad que permite para el consenso y el disenso en el sentido que "toda la política llega a ser comunicación política en el sentido en que la política es constantemente objeto de debates y comunicaciones" (Wolton, 2000, p. 89). Así también lo reafirma Casas (20II), quien dice que la política necesita de dos elementos sustantivos: la información y la comunicación, por lo que su estudio desde esta perspectiva se consolida autónomamente como una ciencia de estudio académico. Abordar a los fenómenos sociales desde la perspectiva teórica de la comunicación permite tener una visión de las transformaciones técnicas, culturales y sociales que se desarrollan a partir de la implementación de los cambios que trae consigo la sociedad de la información.

\section{METODOLOGÍA}

El presente estudio tiene como objetivo hacer una revisión de las formas de abordaje de la comunicación desde las instancias gubernamentales, y comprender los retos y transiciones 
a las que se enfrenta desde la democracia. Para ello se realizó una revisión documental de los enfoques teóricos que estudian la comunicación desde las instituciones públicas, en específico desde los aparatos gubernamentales, centrándose en la comunicación gubernamental y la comunicación pública. La revisión documental "se erige como facilitador del crecimiento intelectual de los individuos en tanto que contribuye a la satisfacción de sus necesidades cognitivas” (Peña y Pirelo, 2007, p. 78). En este sentido, el análisis documental como técnica de recolección de datos permite revisar y analizar los enfoques de la comunicación en las instancias gubernamentales, con la finalidad de generar conocimiento y profundidad de dicha temática.

\section{RESULTADOS}

Antes de comenzar la revisión resulta necesario acotar que el estudio de la comunicación desde las instancias gubernamentales se inserta en el campo de la comunicación política. Esto es porque se comprende a la política como un proceso comunicacional (Meadow, 1980). Dader (2008) reconoce que la ciencia política hace uso de la comunicación sin poder desengancharse de ella, por lo que se encuentra de manera omnipresente en los fenómenos del poder, orden y el conflicto social. De acuerdo con el autor, estos se relacionan con la comunicación en los procesos electorales, la comunicación gubernamental, el escándalo político y la comunicación en crisis, la interacción entre medios de comunicación, periodistas y actores políticos, la ciberdemocracia y democracia electrónica, el humor político y su incidencia en la percepción y reacciones políticas, así como la comunicación política local y los efectos macropolíticos de la imagen política transformada por los medios.

Siguiendo esta categorización, para el estudio de la comunicación desde el gobierno, la comunicación gubernamental se conforma como la vertiente de partida debido a que se constituye desde los aparatos gubernamentales. Desde una perspectiva democrática, la comunicación gubernamental se enmarca en esquemas de representación política en el que "las reglas del juego mediante las cuales unos actores representan a unos sujetos sociales en procura del bienestar común, la cohesión social y el acceso a un futuro cargado de progreso" (Bonilla y García, 1994, p. 69). Desde este enfoque, la comunicación se encuentra auspiciada en planteamiento originario de la "compra" del voto, dejando de lado la visión institucional de legalidad y su regimiento a través de planes rectores para la construcción de las políticas públicas (Canel y Zamora, 2004). En este sentido, esta perspectiva se relaciona con la concepción de una democracia representativa, que sitúa a los ciudadanos como entes votantes, sin que las nociones abarquen una visión más activa de la ciudadanía. 
Por su parte, Canel (1999) entiende a esta disciplina desde la percepción de todos los actores y organizaciones que se comunican de manera estratégica con el fin de convencer, desde el mensaje y su relación con el contexto, y desde la interacción y relación entre los actores involucrados en ella. La comunicación se sitúa en las instituciones; de ahí que sus estudios sean pertinentes para la organización y de los procesos previos a la producción de mensajes políticos de difusión pública (Dader, 2008). Así, la comunicación gubernamental busca la relación con el ciudadano a través de la estrategia para el flujo de información, con el objetivo de que el mayor número de ciudadanos conozcan lo que se proponen (Canel y Zamora, 2004). En ese sentido, la función de informar en los gobiernos se transforma para generar condiciones para el desarrollo de la integración social del colectivo (Tironi y Cavallo, 2008).

Otra visión de la comunicación gubernamental es la de campaña permanente (Ornstein y Mann, 2000). Esta noción concibe a la comunicación de gobierno como una continuación de la campaña electoral, por lo que está basada en la búsqueda continua del apoyo de los electores para mantener el apoyo popular. Canel (1999) menciona que, como consecuencia de la campaña permanente, la comunicación se enfrenta a ciertas tareas: "hacer que el presidente (o el líder gubernamental) sea alguien muy visible, público, que ocupe espacio en los medios de comunicación, de forma que sea claramente reconocible e identificable por los votantes" (p. I03). En ese marco, la comunicación gubernamental tiene la tarea de establecer sensores para detectar necesidades, y mantener como punto clave a su audiencia (Tironi y Cavallo, 2008). Así lo corroboran Gasió y Amadeo (200I), quienes exponen que los gobiernos buscan a través de esta vertiente dar sensación de estabilidad, generar mensajes convincentes, coherentes y compresibles, y hacer de todo ello un proceso vinculado. Eso muestra que la comunicación gubernamental refiere a los actores dentro del poder basado en la publicidad gubernamental, la cual "se constituye como un método a través del cual un gobierno democrático intenta hacer explícitos sus propósitos y orientaciones a un amplio número de personas, para obtener apoyo o consenso en el desarrollo de sus políticas públicas" (Riorda, 20II, p. I06).

Estas concepciones dejan ver también la posibilidad de que la comunicación gubernamental se vincule con las relaciones públicas, derivado de los vínculos que las instituciones gubernamentales tienen que mantener con otros actores, como son los medios de comunicación, para la difusión de sus mensajes. Esto se debe a que la comunicación gubernamental busca la relación con el ciudadano a través de estrategias centradas en el flujo de información, con el objetivo de que el mayor número de ciudadanos conozcan lo que se proponen (Canel y Zamora, 2004). Por ello, la comunicación de las instancias gubernamentales requiere estar en constante pugna con otros actores políticos para "ganar la batalla de la agenda de los medios" (Canel y Sanders, 20io, p. I3). Por tanto, "el uso de las relaciones públicas 
no implica solamente un plan intensivo de emisión de mensajes, sino que, bien entendidas, puedes contribuir al intercambio de diversos públicos” (Amadeo, 20I6, p. I6 ).

Otra visión de abordaje de la comunicación se centra en la premisa de que "la comunicación por naturaleza tiene una función que le es propia: lo político, que es público" (Botero, 2006 , p. I5). Por lo tanto, lo público -como la comunicación y lo político- examina la interacción entre diferentes actores desde los espacios que se configuran para la deliberación pública, por lo que la posibilidad de retomar a la comunicación pública divisa una lógica de comunicación distinta a la gubernamental.

Lo público "se presenta actualmente vinculado a la mediación de los sistemas institucionalizados de comunicación” (De León, 2009, p. I6), enmarcando al Estado como una institución formal. En este sentido, la comunicación pública parte de la perspectiva de que "los 'asuntos públicos' marcan, a su manera, el florecimiento de la democracia en los grupos de interés" (Demers y Lavigne, 2007, p. 69), por lo que se busca un desarrollo deliberativo en el que todos los actores involucrados en ella (gobierno, empresas, medios de comunicación y sociedad civil) "mantengan el mismo reconocimiento de interlocución como configuración ideal” (De León, 2009, p. I6). De esta manera, la comunicación pública contempla a los procesos democráticos en busca de la concertación social a partir del consenso o disenso desde la negociación en colectividad (Botero, 2006).

La comunicación pública refiere a niveles similares para la interlocución entre los actores involucrados, en la que los actores que participan en ella lo hacen "en un contexto de apertura democrática avanzada” (Demers citado en De León, 2009). Por lo tanto, se habla del establecimiento de la interacción entre el gobierno y los ciudadanos (Zémor citado en De León, 2009) que, a su vez, refiere a la apertura a la libertad de opinión, expresión y prensa para hacer posible la crítica pública de las acciones del Estado (Muhlman citado en Demers y Lavigne, 2007).

Desde lo público se permite hablar de democracias participativas en donde se considere al ciudadano como un ente activo, con derecho de visibilidad y un tratamiento mediático de sus opiniones y creencias, a partir "de una sensibilidad acrecentada hacia las formas inéditas del debate social, más allá de los medios de comunicación tradicionales” (Demers y Lavigne, 2007, p. 84). Esto nos permite ver a lo público consolidado y asociado "con una red discursiva en donde se encuentran otros conceptos como la democracia, la participación política, los derechos ciudadanos o la rendición de cuentas” (De León, 2009, p. I7).

En el centro de los cambios de la sociedad de la información, la comunicación pública encuentra también su inserción en las lógicas de interacción que se presentan en Internet. Demers y Lavinge (2007) proponen que los ámbitos de interés de la comunicación pública son la comunicación política, comunicación mediática, las relaciones públicas y la comunicación por redes. De acuerdo con la propuesta, estas se pueden estudiar de manera separada 
o pueden establecer relaciones de estudio entre ellas. En este sentido, se puede observar que los ámbitos de interés son similares a los detectados en la comunicación gubernamental, con excepción de la comunicación por redes, la cual trata del impulso al debate público por parte de los individuos a través de las redes tecnológicas "que pueden llegar a constituir un correlato de las redes sociales” (De León, 2009, p. 23).

En cuanto a los medios de comunicación, Galvis y Botero (2013) consideran que "el peso de la comunicación, cuando es pública, se está tornando cada vez más preponderante, al punto que se ha tornado común que los medios masivos de comunicación tengan que colocar los temas que circulan en el entorno en una agenda pública propia” (p. 28). Desde una perspectiva democrática, los autores consideran a la comunicación pública como "una oportunidad para la construcción democrática de sociedad, a partir de una comunicación estratégica que posibilite escenarios para el desarrollo de comunidades" (Galvis y Botero, 2013, p. 29). Sin embargo, el modelo no se basa en una estrategia hacia medios de comunicación, sino el desarrollo de líneas de acción con la sociedad.

A manera de síntesis, se presenta a continuación las características detectadas para ambos conceptos.

TABLA 1. CARACTERÍSTICAS DE LA COMUNICACIÓN GUBERNAMENTAL Y LA COMUNICACIÓN PÚBLICA

\begin{tabular}{|l|l|}
\hline \multicolumn{1}{|c|}{ COMUNICACIÓN GUBERNAMENTAL } & \multicolumn{1}{c|}{ COMUNICACIÓN PÚBLICA } \\
\hline Basado en la representación política. & Basado en la participación \\
\hline $\begin{array}{l}\text { Situada en las instituciones (comunicación } \\
\text { direccional). }\end{array}$ & $\begin{array}{l}\text { Situada en las instituciones y la sociedad } \\
\text { (comunicación bidireccional). }\end{array}$ \\
\hline $\begin{array}{l}\text { Relación con el ciudadano basada en difusión de } \\
\text { información. }\end{array}$ & Relación con el ciudadano basada en el diálogo. \\
\hline $\begin{array}{l}\text { Vinculada con las relaciones públicas y los medios } \\
\text { de comunicación. }\end{array}$ & $\begin{array}{l}\text { Vinculación directa con el ciudadano y las } \\
\text { organizaciones de la sociedad civil. }\end{array}$ \\
\hline
\end{tabular}

FUENTE: ELABORACIÓN PROPIA.

\section{ANÁLISIS Y DISCUSIÓN}

La comunicación política se encuentra en constante transformación, dando lugar a nuevas prácticas en las que el ciudadano se empodera y logra manifestar abiertamente sus demandas y necesidades, mientras que el aparato gubernamental requiere adaptarse y comenzar procesos 
de innovación que lleven a la transparencia y rendición de cuentas. Desde el carácter institucional, la comunicación política edifica una amplia gama de estudios que no solo van dirigidos a la relación con los medios, sino también al estudio de contextos de acción e interacciones socioculturales entre los productores y los receptores de los contenidos. Bajo el contexto específico descrito, la comunicación política encuentra su punto de convergencia con el ámbito público y el gubernamental, para relacionarse desde diversas formas con el ciudadano.

En la comunicación de los gobiernos, los resultados dejan ver puntos de encuentro entre la comunicación gubernamental y la comunicación pública. Por una parte, se puede precisar que ambos conceptos surgen de su incidencia en lo político como parte de la constitución en espacios democráticos. Sin embargo, tanto lo gubernamental como lo público muestran dos lógicas de comunicación, relacionadas con la democracia representativa y la participativa, respectivamente. Desde la vertiente gubernamental se pretende dar a conocer la información que el estado genera a través de sus lineamientos y acciones, por lo que coloca en un rol central a las acciones del gobierno para mantener a la ciudadanía como una audiencia con ciertos intereses y necesidades detectables desde lo masivo, que requieren ser atendidos para mantener su adhesión y apoyo a cierta filosofía o partido político. Esto refiere a que la comunicación gubernamental se mantiene dentro de un esquema de comunicación unidireccional, en el que se establece una estructura vertical del poder para la emisión de los mensajes hacia el ciudadano. Sus derivaciones como campaña permanente y su relación con las relaciones públicas muestran una visión corporativa de los aparatos gubernamentales.

Por otro parte, desde lo público, se sostiene un lógica comunicativa de circulación de la información y de opiniones, lo que conlleva a sostener regímenes flexibles y abiertos, así como una estructura de similitud de oportunidades para la emisión de los mensajes (estructura horizontal), en la que el papel del Estado solo es "un actor más, y a menudo uno contra el que los actores se asocian” (Demers y Lavigne, 2007, p. 77). Por tanto, no se trata únicamente de la difusión de los mensajes políticos a través de los canales de comunicación, sino que implica aceptar lo que los ciudadanos tengan que decir a través de estos medios para así generar consensos. En un esquema público de la comunicación, las lógicas de comunicación establecen una igualdad en las posibilidades de comunicación. En este sentido, se puede comprender que la comunicación pública tiene una perspectiva similar a los procesos intersubjetivos, ya que se centra en la construcción de consensos. Es así como su provecho viene de las combinaciones de valores y resultados técnicos, así como de ideales e intereses, de manera que su relación con el gobierno abierto se percibe desde el fomento que Internet predica hacia las formas colaborativas y la comunicación uno a uno. El esquema de la comunicación pública permite ver su afinidad con los esquemas de una democracia participativa, que consiente un mayor involucramiento de la sociedad en los asuntos públicos. 


\section{CONCLUSIONES}

Lo hasta ahora abordado lleva a afirmar que, dentro de la sociedad actual, la comunicación se supone más abierta y, por tanto, más transparente y próxima. Bajo el panorama de iniciativas que buscan la apertura gubernamental, los puntos de encuentro entre la comunicación, tecnología y gobierno llevan a considerar a la comunicación pública como la lógica que permite un mayor acercamiento entre los ciudadanos y sus representantes políticos. No se trata de negar la existencia de la comunicación gubernamental y la comunicación pública en el mismo entorno, sino de crear un espacio de convergencia en el que las instancias gubernamentales procuren sus actividades comunicativas hacia el exterior, pero que también permitan dar paso a que la sociedad incida en las decisiones públicas. Así, la comunicación gubernamental y la comunicación pública deben ser elementos presentes en la gestión gubernamental. Si se afronta bajo el anclaje de la comunicación gubernamental, se trataría a la comunicación como una estructura vertical centrada en la emisión de mensajes unidireccionales (gobierno-ciudadano). Mientras que desde lo público se permite hablar de democracias participativas en donde se considere al ciudadano como un ente activo, con derecho de visibilidad y un tratamiento mediático de sus opiniones y creencias.

En este sentido, el contexto define que ante los cambios que trae consigo la democracia participativa los procesos de socialización adquieren mayor relevancia, al dar un mayor reconocimiento a los ciudadanos y reconocer sus distintas culturas y perspectivas. No es posible sostener, entonces, las formas tradicionales de interacción entre el ciudadano y el gobierno, por lo que el progreso se convierte en clave para evitar sistemas hegemónicos y dominantes. Por tanto, en el marco de una sociedad tecnologizada la comunicación pública se redefine a partir de la presencia de Internet; es así como su provecho viene de las combinaciones de valores y resultados técnicos, así como de ideales e intereses, de manera que su relación con el gobierno abierto se percibe desde el fomento de formas colaborativas y la comunicación uno a uno.

La democracia participativa, entonces, permite abrir un espacio para lógicas de comunicación que suponen cambiar los esquemas tradicionalmente verticales, hacia una apertura al diálogo, lo que conlleva a cambios en las representaciones simbólicas que se tienen de la relación entre ciudadanos y servidores públicos. Esto conlleva a visualizar que la comunicación pública contiene rasgos intersubjetivos que permiten reconocer la construcción de consensos, así como los elementos que facilitan u obstaculizan el diálogo. En este sentido, tanto los ciudadanos como gobernantes son participantes del espacio público; de esta manera, se reconoce a los ciudadanos como públicos que no son pasivos, sino que valoran los asuntos políticos a través de la resolución de problemas y la presencia mediática. 
Afrontar los cambios en la comunicación desde los gobiernos supone apegarse a la transición democrática y adoptar sus elementos constitutivos para incorporarlos en la forma de comunicare con los ciudadanos. Los cambios en dichos esquemas institucionales comunicativos reflejan un cambio en la interacción y abren la posibilidad de nuevas representaciones simbólicas de la política, transformaciones en las relaciones de poder, así como la posibilidad de crear nuevas actitudes y procesos de interpretación en torno a la acción comunicativa con el otro.

\section{REFERENCIAS}

Aguirre, J. (20I2). La participación ciudadana y las redes sociales. En Décimo tercer certamen de ensayo político (pp. 73-94). CEE. http://www.cee-nl.org.mx/educacion/certamen_ensayo/XIIICDEP_MH_Laparticipacion.pdf

Amadeo, B. (2016). El estudio de la comunicación gubernamental: líneas de investigación y futuros desafíos. Austral Comunicación, 5(2), I55-I8I.

Bonilla, J.y García, E. (1994). Nuevas dinámicas de representación política:movimientos sociales, espacio público y redes de comunicación. Signo y pensamiento, 13(25), 67-82.

Botero, L. (2006). Comunicación pública, comunicación política y democracia: un cruce de caminos. Anagramas, 5(9), 13-27. http://www.redalyc.org/pdf/649/64900202.pdf

Canel, M. (1999). Comunicación política: técnicas y estrategias para la sociedad de la información. Tecnos.

Canel, M. y Sanders, K. (2010). Para estudiar la comunicación de los gobiernos: un análisis del estado de la cuestión. Comunicación y Sociedad, 23(I), 7-48.

Canel, M. y Zamora, R. (2004). La comunicación en las organizaciones políticas: la estrategia permanente en el mercado de la visibilidad de los poderes público. En J. Losada, Gestión de comunicación en las organizaciones (págs. 515-542). Ariel.

Casas, M. (20II). Medios de comunicaciònn, nuevas tecnologías y el futuro de la política. En C. Muñiz, Comunicación, política y ciudadanía. Aportaciones actuales al estudio de la comunicacion política. (págs. 79-102). Fontamara.

Castells, M. (1997, 25 de octubre). ¿Fin del Estado nación? El país. https://elpais.com/diario/1997/10/26/opinion/877816803_850215.html

Dader, J. (2008). La adolescente investigación en Comunicación Política: Estructura del campo y tendencias prometedoras. En M. Martínez Nicolás, Para investigar la comunicación. Propuestas teórico-metodológicas. Tecnos.

Demers, F. y Lavigne, A. (2007). La comunicación pública: una prioridad contemporánea de la investigación. Comunicación y Sociedad (7), 65-87.

Foucault, M. (1979). Microfisica del poder. Edissa.

Galvis, C. y Botero, L. (2013). Comunicación pública de la ciencia como factor de consolidación democrática. Redes.com (7), 25-41. https://dialnet.unirioja.es/descarga/articulo/44969I2.pdf 
Gasió, G. y Amadeo, B. (200I). Notas sobre una experiencia de comunicación gubernamental: el Ministerio de Economía argentino, I99I-I996. En O. De Masi, Comunicación gubernamental (págs. 33-6o). Paidós.

González, E. (2008). El perfil del ciudadano en una democracia:: Gobierno del Estado de Querétaro. Habermas, J. (1994). Historia y crítica de la opinión pública. G. Gili.

Meadow, R. (1980). Politics as Communication. Ablex Publishing.

Mill, J. S. (I86I). Representative Government. Batoche Books.

Monterroso, N. (2009). El estudio del desarrollo. Etapas y paradigmas teóricos. Universidad Autónoma del Estado de México.

O'Reilly, T. (2005, 30 de septiembre). O'Reilly. What is Web 2.o. Design patterns and business models for next generation of software: http:/www.oreilly.com/pub/a/web2/archive/what-isweb-20.html

Ornstein, N., y Mann, T. (2000). The permanent campaign and its future. American Enterprise Institute.

Pateman, C. (1970). Participation and Democratic Theory. Cambridge University Press.

Peña, T. y Pirelo, J. (2007). La complejidad del análisis documental. Información, cultura y sociedad: revista del Instituto de Investigaciones Bibliotecológicas (I6), 5581. https://www.redalyc.org/ pdf/2630/263019682004.pdf

Rawls, J. (1971). A theory of Fustice. The Belknap Press of Harvard University Press.

Riorda, M. (20II). La comunicación gubernamental como comunicación gubernamental. Politai. (3)2, 96-III. https://revistas.pucp.edu.pe/index.php/politai/article/view/13956

Sartori, G. (1993). ¿Qué es la democracia? Editorial Patria.

Schumpeter, J. (1943). Capitalism, Socialism and Democracy. Allen \& Unwin.

Serrano, A. (2015). La participación ciudadana en México. Estudios Políticos(34), 93-II6.

Subirats, J. (2007). Democracia, participación ciudadana y transformación social. En I. Ahedo y P. Ibarra, Democracia participativa y desarrollo bumano (págs. 23-36). Dykinson.

Tironi, E. y Cavallo, A. (2008). Comunicación estratégica. Vivir en un mundo de señales. Taurus.

Villodre, J. (20I9). Innovación pública abierta. Eunomía. Revista en Cultura de la Legalidad (17), 3I4327. https://doi.org/10.20318/eunomia.2019.5036

Wolton, D. (2000).Internet, ¿Y después?. Una teoría crítica de los nuevos medios de comunicación. Gedisa. Zallo, R. (20I6). Tendencias en comunicación. Cultura digital y poder. Gedisa.

Esta obra está bajo Licencia Creative Commons Atribución-NoComercial-SinDerivadas 4.0 Internacional. 